\title{
Modeling and Analysis of Population Dynamics of Human Cells Pertaining to HIVIAIDS with Treatment
}

\author{
Kumama Regassa, Purnachandra Rao Koya \\ Department of Mathematics, Wollega University, Nekemte, Ethiopia \\ Email address: \\ Kumamaregassa@gmail.com (K. Regassa),drkpraophd@gmail.com (P. R. Koya) \\ To cite this article: \\ Kumama Regassa, Purnachandra Rao Koya. Modeling and Analysis of Population Dynamics of Human Cells Pertaining to HIV/AIDS with \\ Treatment. American Journal of Applied Mathematics. Vol. 7, No. 4, 2019, pp. 127-136. doi: 10.11648/j.ajam.20190704.14
}

Received: June 28, 2019; Accepted: August 3, 2019; Published: September 20, 2019

\begin{abstract}
In this paper, a mathematical model has been formulated to describe the population dynamics of human cells pertaining to the HIV/AIDS disease with ART as treatment and is analyzed. The human cells have been divided into four compartments Susceptible - Asymptomatic - Symptomatic - AIDS (SAIV). The well posedness of the four dimensional dynamical system is proved and the steady states of the model are identified. Additionally, parametric expression for the basic reproduction number is constructed following next generation matrix method and analyzed its stability using Routh Hurwitz criterion. From the analytical and numerical simulation studies it is observed that if the basic reproduction is less than one unit then the solution converges to the disease free steady state i.e., disease will wipe out and thus the treatment is said to be successful. On the other hand, if the basic reproduction number is greater than one then the solution converges to endemic equilibrium point and thus the infectious cells continue to replicate i.e., disease will persist and thus the treatment is said to be unsuccessful. Sensitivity analysis of the model parameters is conducted and their impact on the reproduction number is analyzed. Finally, the model of the present study simulated using MATLAB. The results and observations have been included in the text of this paper lucidly.
\end{abstract}

Keywords: HIV, ART, Basic Reproduction Number, Stability Analysis, Routh Hurwitz Criterion

\section{Introduction}

The Human Immunodeficiency Virus HIV infects cells of the immune system as well as that of the central nervous system inhuman body. The T-helper lymphocytes are the main type of cells that will be infected by HIV disease. It is well known that the role of these T-helper lymphocytes cells in the immune system is to coordinate with the actions of other immune system cells. A large reduction in the number of these cells results in weakening the immune system $[1,2]$.

HIV infects the T-helper cells because it has the protein called CD4+ on its surface, which HIV uses to attach itself to cells before entering to them. That is why the T-helper cell is referred to as a CD4+T lymphocyte. Once it attaches itself into a cell, HIV produces new copies which are capable of infecting other cells.

According to WHO clinical staging of HIV/AIDS, HIV infection has four distinct stages viz., acute stage, Asymptomatic stage, Asymptomatic stage and Advanced AIDS stage [3]. These four stages of the disease have been introduced in what follows.

Stage 1 (Primary HIV infection): First stage of HIV infection is called primary infection stage. Primary infection begins shortly after an individual becomes infected with HIV for the first time. This stage lasts for a few weeks. During this period, individuals experience Flu like symptoms. Very few individuals seek treatment during this stage and those are usually misdiagnosed as if they are suffering from general viral infection. It is common that whenever an HIV test is performed in a medical lab then the result may come out to be negative. The reason for such negative results is antibodies which are yet not produced by the individual's immune system. Since antibodies have not yet been developed, HIV continues to replicate, resulting in a very high level of the virus [4]. Few weeks after getting the infection, the infected individuals become highly infectious. At this stage there would be a large amount of HIV in the peripheral blood amounting around 106 copies of virus per micro-litter $\mu$ l of blood. Peripheral blood is the blood which is in the circulating system but not in the lymphatic system, 
bone marrow, liver. Antibodies and cytotoxic lymphocyte start getting produced in response to the virus which is known as sero-conversion. At this stage about 20 percent of people who are HIV positive show symptoms which are not mild. However, any diagnosis for detecting HIV infection at this stage will go wrong as already mentioned. Those who believe that they were exposed to HIV should repeat the medical test after six months.

Stage 2 (Asymptomatic HIV): In the second stage, individuals become free from all types of symptoms of HIV although there will be some swollen glands. The HIV appears in blood in a very low Level, but is detectable. If an HIV test is performed, the result will come out to be positive. While the individuals remain asymptomatic, the HIV in their blood continues to reproduce constantly. This stage lasts for about ten years. However, the period of second stage can be much longer or shorter depending on the individual and is also characterized by a CD4+count whose normal count is around 500 cells per $\mu 1$.

Stage 3 Symptomatic HIV: In the third stage, symptoms start appearing and the immune system becomes so damaged by HIV. Further, it leads to greater destruction ofCD4+ cell and the immune system will not able to replace them. By now the immune system fails and as a result the symptoms start developing [3]. Symptoms are typically mild initially and they gradually become more severe. Opportunistic infections, infections that take advantage of the vulnerable immune system, begin to occur. These infections affect almost all the systems of the body and include both infections and cancers. Some common opportunistic infections include tuberculosis, cytomegalovirus, and shingles. In this stage HIV infection is often characterized by multi-system diseases and infections in body. Treatment for a particular infection or cancer is often carried out. However, the main cause is the action of HIV as it attacks the immune system. Unless HIV is reduced the immune suppression will continue to be weaker.

Stage 4 (Acquired Immune Deficiency Syndrome AIDS): In fourth and last stage, a person can be medically tested positive as having AIDS. The progression to AIDS can be characterized by CD4+ count which is 200 per $\mathrm{ml}$ or below in a patient, while it is around 1000 per $\mathrm{ml}$ in a normal person. At this stage, the infected individual is likely to develop opportunistic infections in their respiratory system, gastro-intestinal system, central nervous system and on the skin as well. Once a person is diagnosed with AIDS, the AIDS status is permanent. A blood test can determine if a person is infected with HIV, but if a person tests positive for HIV, it does not necessarily mean that the person has AIDS. A diagnosis of AIDS is made by a physician according to the CDC AIDS Case definition.

Organization of the paper: In Section 2, assumptions of the model are stated and based on which a mathematical model for describing the population dynamics of human body cells pertaining to the HIV/AIDS disease is formulated.

In section 3 , well possedness of the model formulation, stability analysis of the equilibrium points and reproduction number are included.

In Section 4, numerical simulation studies of the model equations are performed by assigning various sets of numerical values to the model parameters.

In Section 5 sensitivity analysis of model parameters towards the reproduction number is carried out.

In section 6 Result and Discussion are presented. Finally in Section 7, conclusions are stated.

\section{Model Formulation}

In the present model describing Human Immunodeficiency virus (HIV) with treatment the total human body cells are divided into four classes: (i) Susceptible cells class. It is denoted by $S(t)$. These susceptible cells are capable of becoming infected cells in future. These include new cells that not infected yet. (ii) Asymptomatic cells class. It is denoted by $A(t)$. This class consists of cells which are infected with virus but shows no signs of infections. These cells are active to transmit infections to other cells but they still continue as normal. (iii) Symptomatic cells class. It is denoted by $I(t)$. This class consists of infectious cells and they show signs of infections. Such cells manifest their weakness as they harmed by virus. (iv) AIDS cells class. It is denoted by $V(t)$. They are cells that highly contains virus that weakens T-helper cells. This categorical cell requires high medications and cares.

Here, a mathematical model of the Human Immunodeficiency virus (HIV) is constructed based on the following assumptions made on the human body cells:

i. The total population size of human body cells is assumed to be constant.

ii. Both the numbers of births and deaths of cells are equal.

iii. Human Immunodeficiency virus (HIV) model classifies the cell population into four compartments SAIV at any time.

iv. Susceptible cells are recruited into the compartment $S(t)$ at a constant rate $\tau$.

v. Susceptible cells are infected when they come into effective contact with asymptomatic cells and join asymptomatic cells class at a rate $\beta$.

vi. Asymptomatic cells join symptomatic cells at a rate $\alpha$ and AIDS cells at arate $\delta$.

vii. The symptomatic cells join AIDS cells class at a rate $\omega$.

viii. All types of cells suffer natural mortality with a rate $\mu$.

ix. AIDS cells die of infection at a rate $\gamma$.

x. Symptomatic cells die due at the rate of $\rho$ due to disease.

xi. Asymptomatic cells die due to disease at the rate of $\rho$. xii. Asymptomatic and Symptomatic cells are treated All parameters used in the model are positive.

Table 1. Notations and description of model variables.

\begin{tabular}{ll}
\hline Variable & Description \\
\hline $\mathrm{S}(\mathrm{t})$ & Population size of susceptible cells \\
$\mathrm{A}(\mathrm{t})$ & Population size of asymptomatic cells \\
$\mathrm{I}(\mathrm{t})$ & Population size of symptomatic cells \\
$\mathrm{V}(\mathrm{t})$ & Population size of AIDS cells \\
\hline
\end{tabular}


Table 2. Notations and description of model parameters.

\begin{tabular}{ll}
\hline Parameter & Description \\
\hline$\tau$ & Recruitment rate of susceptible cells. With this rate new cells will born and they will enter into susceptible class. \\
$\beta$ & Transmission rate of infection cells. With this rate cells transfer from compartment $S$ to $A$. \\
$\alpha$ & Rate of cells transferring from compartment $A$ to $I$. \\
$\omega$ & Rate of cells transferring from compartment $I$ to $V$. \\
$\phi$ & Treatment rate of $I$. \\
$\gamma$ & Death rate of cells due to infection. With this rate cells of $V$ compartment die of the disease. \\
$\mu$ & Natural death rate. With this rate cells of all compartments die naturally. \\
$\eta$ & Treatment rate of asymptomatic cells $I$. \\
$\rho$ & Death rate of symptomatic cells $I$ due to disease. \\
\hline
\end{tabular}

Based on the basic assumptions together with the description of both model variables and parameters the schematic diagram of the model compartments and the cell flow directions can be given as in Figure 1.

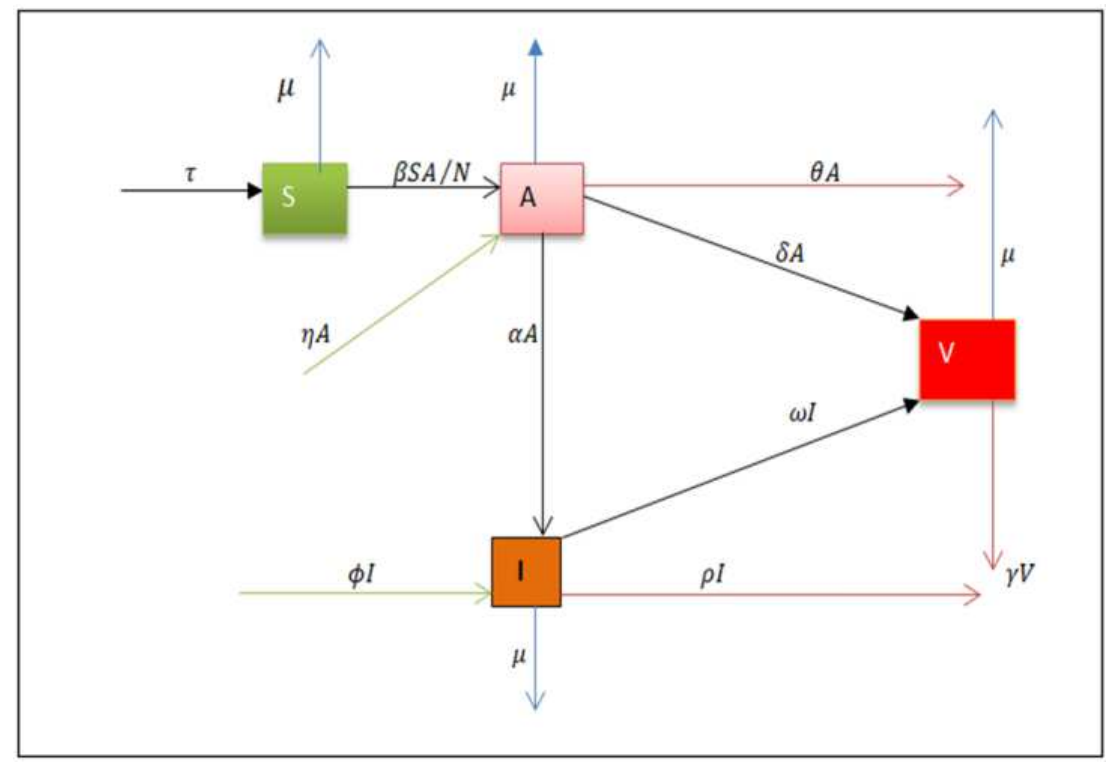

Figure 1. Schematic diagram of compartmental structure of the model.

Based on the model assumptions, the notations of variables and parameters and the schematic diagram, the model equations are formulated and are given as follows:

$$
\begin{gathered}
d S / d t=\tau-(\beta A S / N)-\mu S \\
d A / d t=(\beta A S / N)-(\alpha+\mu+\theta+\delta-\eta) A \\
d I / d t=\alpha A-(\omega+\mu+\rho-\emptyset) I \\
d V / d t=\delta A+\omega I-(\mu+\gamma) V
\end{gathered}
$$

The non-negative initial conditions of the model equations (1) - (4) are denoted by $S(0)>0, A(0) \geq 0, I(0) \geq$ $0, V(0) \geq 0$. This system consists of four first order nonlinear ordinary differential equations.

\section{Mathematical Analysis of the Model}

In this section mathematical analysis of the present improved and modified model is conducted. The analysis consists of the features including (i) existence, positivity and boundedness of solutions (ii) steady states (iii) disease free equilibrium points (iv) endemic equilibrium points (v) basic reproduction number (vi) stability analysis of the disease free equilibrium points (vii) local stability of disease free equilibrium point (viii) global stability of disease free equilibrium point (ix) stability analysis of endemic equilibrium point and (x) local stability of endemic equilibrium point. These mathematical aspects of the model are presented and discussed in the following sub-sections respectively.

\subsection{Existence, Positivity and Boundedness of Solution}

In order to show that the model is biologically valid, it is required to prove that the solutions of the system of differential equations (1) - (4) exist and are both positive and bounded for all time. It is done starting with proving Lemma 1.

Lemma 1 (Positivity) Solutions of the model equations (1) - (4) together with the initial conditions $S(0) \geq 0, A(0) \geq$ $0, I(0) \geq 0, V(0) \geq 0$ are always positive (OR) the model variables $S(t), A(t), I(t)$, and $V(t)$ are positive for all $t$ and will remain in $\mathbb{R}_{+}^{4}$.

Proof Positivity of the solutions of model equations is shown separately for each of the model variables 
$S(t), A(t), I(t)$, and $V(t)$.

Positivity of $S(t)$ : The model equation (1) given by $d S / d t=\tau-(\beta A S / N)-\mu S$ can be expressed without loss of generality, after eliminating the positive term $\tau$ appearing on the right hand side, as an inequality as $d S / d t \geq$ $-[\mu+(\beta A / N)] S$. Using variables separable method and on applying integration, the solution of the foregoing differentially inequality can be obtained as $S(t) \geq$ $S(0) e^{-\mu t-\beta \int A(t) d t}$. Recall that an exponential function is always non-negative irrespective of the sign of the exponent i.e. the exponential function $e^{-\mu t-\beta \int A(t) d t}$ is a non-negative quantity. Hence, it can be concluded that $S(t) \geq 0$.

Positivity of $A(t)$ : The model equation (2) given by $d A / d t=(\beta A S / N)-(\alpha+\mu+\theta+\delta-\eta) A \quad$ can $\quad$ be expressed without loss of generality, after eliminating positive term $(\beta A S / N)$ and $\eta A$ which are appearing on the right hand side, as an inequality as $d A / d t \geq-(\alpha+\mu+\theta+$ $\delta) A$. Using variables separable method and on applying integration, the solution of the foregoing differentially inequality can be obtained as $A(t) \geq A(0) e^{-(\alpha+\mu+\theta+\delta) t}$. Recall that an exponential function is always non-negative irrespective of the sign of the exponent i.e. the exponential function $e^{-(\alpha+\mu+\theta+\delta) t}$ is a non-negative quantity. Hence, it can be concluded that $A(t) \geq 0$.

Positivity of $I(t)$ : The model equation (3) given by $d I / d t=\alpha A-(\omega+\mu+\rho-\emptyset) I$ can be expressed without loss of generality, after eliminating the positive terms $(\alpha A)$ and $(\phi I)$ which are appearing on the right hand side, as an inequality as $d I / d t \geq-(\omega+\mu+\rho) I$. Using variables separable method and on applying integration, the solution of the foregoing differentially inequality can be obtained as $I(t) \geq I(0) e^{-(\omega+\mu+\rho) t}$. Recall that an exponential function is always non-negative irrespective of the sign of the exponent i.e. the exponential function $e^{-(\omega+\mu+\rho) t}$ is a non-negative quantity. Hence, it can be concluded that $I(t) \geq 0$.

Positivity of $V(t)$ : The model equation (4) given by $d V / d t=\delta V+\omega I-(\mu+\gamma) V$ can be expressed without loss of generality, after eliminating the positive terms $\delta V, \omega I$, and $\gamma V$ which are appearing on the right hand side, as an inequality as $d V / d t \geq-(\mu+\gamma) V$. Using variables separable method and on applying integration, the solution of the foregoing differentially inequality can be obtained as $V(t) \geq V(0) e^{-(\mu+\gamma) t}$. Recall that an exponential function is always non-negative irrespective of the sign of the exponent i.e. the exponential function $e^{-(\mu+\gamma) t}$ is a non-negative quantity. Hence, it can be concluded that $V(t) \geq 0$.

Thus, the model variables $S(t), A(t), I(t)$, and $V(t)$ representing population sizes of various types of human body cells are positive quantities and will remain in $\mathbb{R}_{+}^{4}$ for allt.

Lemma 2 (Boundedness) The positive solutions of the system of model equations (1) - (4) are bounded. That is the model variables $S(t), A(t), I(t)$ and $V(t)$ are all bounded for all $t$.

Proof: Recall that each population size is bounded if and only if the total population size is bounded. Hence, in the present case it is sufficient to prove that the total population size $N=S(t)+A(t)+I(t)+V(t)$ is bounded for all $t$. It can be begun by showing that all feasible solutions are uniformly bounded in a proper subset $\Omega \in \mathbb{R}_{+}^{4}$ where the feasible region $\Omega$ is given by $\Omega=\{(S, A, I, V) \in$ $\left.\mathbb{R}_{+}^{4} ; \mathrm{N} \leq[\tau /(\mu-\eta-\phi)]\right\}$.

Now, summation of all the four equations (1) - (4) of the model gives $d N(t) / d t=\tau+\eta A+\phi I-\mu N(t)-\theta A-\rho I-$ $\gamma \mathrm{V}$. It can be expressed without loss of generality, after eliminating the negative terms $(-\theta \mathrm{A}-\rho \mathrm{I}-\gamma \mathrm{V})$ which are appearing on the right hand side, as an inequality as $\mathrm{dN}(\mathrm{t}) / \mathrm{dt} \leq[\pi+(\eta+\phi-\mu) \mathrm{N}(\mathrm{t})]$. Equivalently this inequality can be expressed as a linear ordinary differential inequality as $[\mathrm{dN}(\mathrm{t}) / \mathrm{dt}]+[(\eta+\phi-\mu) \mathrm{N}(\mathrm{t})] \leq \tau$ giving general solution upon solving as $\mathrm{N}(\mathrm{t}) \leq[\tau /(\mu-\eta-\phi)]+$ $c e^{-(\mu-\eta-\phi) t}$. But, the term $N(0)$ denotes the initial values of the respective variable i.e. $\mathrm{N}(\mathrm{t})=\mathrm{N}(0)$ at $\mathrm{t}=0$. Thus, in terms of a new parameter $\mu_{d}=(\mu-\eta-\phi)>0$, the particular solution can be expressed as $\mathrm{N}(\mathrm{t}) \leq\left(\tau / \mu_{d}\right)+$ $\left[\mathrm{N}(0)-\left(\tau / \mu_{d}\right)\right] e^{-\left(\tau / \mu_{d}\right) t}$. Further, it can be observed that $N(t) \rightarrow\left(\tau / \mu_{d}\right)$ as $t \rightarrow \infty$. That is, total population size $N(t)$ takes off from a value $\mathrm{N}(0)$ at the initial time $\mathrm{t}=0$ and ends up with a bounded value $\left(\tau / \mu_{d}\right)$ as the time $t$ progresses to infinity. Thus, it can be concluded that $N(t)$ is bounded as $0 \leq N(t) \leq\left(\tau / \mu_{d}\right)$.

Therefore, $\left(\pi / \mu_{d}\right)$ where $\mu_{d}=(\mu-\eta-\phi)$ is an upper bound of $N(t)$. Hence, feasible solution of the system of model equations (1) - (4) remains in the region $\Omega$ which is positively invariant set. Thus, the system is biologically meaningful and mathematically well posed in the domain $\Omega$. Further, it is sufficient to consider the dynamics of the populations represented by the model system (1) - (4) in that domain.

Therefore, it can be summarized the result of Lemma 2 as "the model variables $S(t), A(t), I(t)$, and $V(t)$ are bounded for all $t$ ". Also, here Lemma 2 sets a restriction on the model parameters as $(\mu-\eta-\phi)>0$ or equivalently $\mu>(\eta+\phi)$.

Lemma 3 (Existence) Solutions of the model equations (1) - (4) together with the initial conditions $S(0)>0, A(0) \geq$ $0, I(0) \geq 0, V(0) \geq 0$ exist in $\mathbb{R}_{+}^{4}$ i.e. the model variables $S(t), A(t), I(t)$, and $V(t)$ exist for all $t$ and will remain in $\mathbb{R}_{+}^{4}$.

Proof: Let the right hand sides of the system of equations (1) - (4) are expressed as follows:

$$
\begin{gathered}
d S / d t=\tau-(\beta A S / N)-\mu S \equiv g_{1} \\
d A / d t=(\beta A S / N)-(\alpha+\mu+\theta+\delta-\eta) A \equiv g_{2} \\
d I / d t=\alpha A-(\omega+\mu+\rho-\emptyset) I \equiv g_{3} \\
d V / d t=\delta A+\omega I-(\mu+\gamma) V \equiv g_{4}
\end{gathered}
$$

According to Derrick and Groosman theorem, let $\Omega$ denote the region $\Omega=\left\{(S, A, \quad I, \quad V) \in \mathbb{R}_{+}^{4} ; \mathrm{N} \leq\left(\pi / \mu_{d}\right)\right\}$. Then equations (1) - (4) have a unique solution if $\left(\partial f_{i}\right) /\left(\partial x_{j}\right): i, j=1,2,3,4$ are continuous and bounded in $\Omega$. Here, $x_{1}=S, x_{2}=A, x_{3}=I, x_{4}=V$.

The continuity and the boundedness of $g_{1}, g_{2}, g_{3}, g_{4}$ are verified as here under:

For $g_{1}$ :

$$
\left|\left(\partial g_{1}\right) /(\partial S)\right|=|-[\mu+\beta A / N]|<\infty
$$




$$
\begin{gathered}
\left|\left(\partial g_{1}\right) /(\partial A)\right|=|-\beta S / N|<\infty \\
\left|\left(\partial g_{1}\right) /(\partial I)\right|=0<\infty \\
\left|\left(\partial g_{1}\right) /(\partial V)\right|=0<\infty
\end{gathered}
$$

For $g_{2}$ :

$$
\begin{gathered}
\left|\left(\partial g_{2}\right) /(\partial S)\right|=|\beta A / N|<\infty \\
\left|\left(\partial g_{2}\right) /(\partial A)\right|=|\beta S / N-(\alpha+\theta+\mu+\delta-\eta)|<\infty \\
\left|\left(\partial g_{2}\right) /(\partial I)\right|=0<\infty \\
\left|\left(\partial g_{2}\right) /(\partial V)\right|=0<\infty
\end{gathered}
$$

For $g_{3}$ :

$$
\begin{gathered}
\left|\left(\partial g_{3}\right) /(\partial S)\right|=0<\infty \\
\left|\left(\partial g_{3}\right) /(\partial A)\right|=|\alpha|<\infty \\
\left|\left(\partial g_{3}\right) /(\partial I)\right|=|-(\omega+\rho+\mu-\phi)|<\infty \\
\left|\left(\partial g_{3}\right) /(\partial V)\right|=0<\infty
\end{gathered}
$$

For $g_{4}$ :

$$
\begin{gathered}
\left|\left(\partial g_{4}\right) /(\partial S)\right|=0<\infty \\
\left|\left(\partial g_{4}\right) /(\partial A)\right|=|\delta|<\infty \\
\left|\left(\partial g_{4}\right) /(\partial I)\right|=|\omega|<\infty \\
\left|\left(\partial g_{4}\right) /(\partial V)\right|=|-(\mu+\gamma)|<\infty
\end{gathered}
$$

Thus, all the partial derivatives $\left(\partial f_{i}\right) /\left(\partial x_{j}\right): i, j=$ $1,2,3,4$ exist, continuous and bounded in $\Omega$. Hence, by Derrick and Groosman theorem, a solution for the model (1) - (4) exists and is unique.

\subsection{Steady State Solutions}

In order to understand the dynamics of the model, it is necessary to determine equilibrium points of the solution region. An equilibrium solution is a steady state solution of the model equations (1) - (4) in the sense that if the system begins at such a state, it will remain there for all times. In other words, the population sizes remain unchanged and thus the rate of change for each population vanishes. Equilibrium points of the model are found, categorized, stability analysis is conducted and the results have been presented in the following sub-sections:

\subsubsection{Disease Free Equilibrium Point}

Disease free equilibrium point is a steady state solution where there is no disease in the population. Now, absence of disease implies that $A(t)=I(t)=V(t)=0$ and also setting the right hand sides of the model equations equal to zero results in giving

$$
\tau-\mu S=0
$$

Solutions of which is the population size of the susceptible humans at the disease free equilibrium and is given by

$$
S^{0}=(\tau / \mu)
$$

Thus, the disease free equilibrium point of the model equations (1) - (4) is given by

$$
E_{0}=\left\{S^{0}, I^{0}, \quad A^{0}, \quad V^{0}\right\}=\{\tau / \mu, 0, \quad 0,0\}
$$

\subsubsection{Endemic Equilibrium Point}

The endemic equilibrium point $E_{1}=\left\{\begin{array}{llll}S^{1}, & I^{1}, & A^{1}, & V^{1}\end{array}\right\}$ is a steady state solution when the disease persists in the population. The endemic equilibrium point is obtained by setting rates of changes of variables with respect to time of model equations (1) - (4) to zero. That is, setting $d S / d t=$ $d A / d t=d I / d t=d V / d t=0$ the model equations take the form as

$$
\begin{gathered}
\tau-(\beta A S / N)-\mu S=0 \\
(\beta A S / N)-a A=0 \\
\alpha A-b I=0 \\
\delta A+\omega I-c V=0
\end{gathered}
$$

Here in (5) - (8), the quantities $a, b, c$ represent the parametric expressions as $a=\alpha+\mu+\theta+\delta-\eta, b=\omega+$ $\mu+\rho-\emptyset, c=\mu+\gamma$. Clearly, solutions of (5) - (8) will provide endemic equilibrium of the model equations and that is obtained as follows:

Now, (6) can be rearranged as $[(\beta S / N)-a] A=0$ leading to the solutions $(\beta S / N)-a=0$ or $A=0$ or both. However, $A$ does not vanish since the disease is assumed to persist. Thus, it leads to the only meaningful solution $(\beta S / N)-a=$ 0 or equivalently $S=(a N / \beta)$. That is, the $S^{1}$ component of $E_{1}$ is given by

$$
S^{1}=(a N / \beta)=\left(a \tau / \beta u_{d}\right)=\left(\tau / \mu R_{0}\right)
$$

Similarly, solving (7) and (8) gives expression for $I$ and $V$ as

$$
\begin{gathered}
I=(\alpha A / b) \\
V=(\delta A / c)+(\omega I / c)
\end{gathered}
$$

Further, substitution of equation (9) into (5) gives $[\tau-$ $\beta(a / \beta)(A)-\mu(a / \beta)]=0$. But since $N=\left(\pi / \mu_{d}\right)$ and after some algebraic simplifications, an expression for $A$ can be obtained as

$$
A^{1}=\left(\mu \tau / \beta \mu_{d}\right)\left(R_{0}-1\right)
$$

Finally, substitution of $A^{1}$ into (10) and (11) respectively gives the expressions for $I$ and $V$ in terms of parameters as

$$
\begin{gathered}
I^{1}=\left(\alpha \mu \tau / b \beta \mu_{d}\right)\left(R_{0}-1\right) \\
V^{1}=\left(\mu \tau / \beta \mu_{d}\right)[(\delta / c)+(\omega \alpha / c b)]\left(R_{0}-1\right)
\end{gathered}
$$

Therefore, the endemic equilibrium point is given by $E_{1}=\left\{S^{1}, I^{1}, A^{1}, V^{1}\right\}$ where

$$
S^{1}=\left(a \tau / \beta u_{d}\right)=\left(\tau / \mu R_{0}\right)
$$




$$
\begin{gathered}
A^{1}=\left(\mu \tau / \beta \mu_{d}\right)\left(R_{0}-1\right) \\
I^{1}=\left(\alpha \mu \tau / b \beta \mu_{d}\right)\left(R_{0}-1\right) \\
V^{1}=\left(\mu \tau / \beta \mu_{d}\right)[(\delta / c)+(\omega \alpha / c b)]\left(R_{0}-1\right)
\end{gathered}
$$

\subsection{Basic Reproduction Number}

The basic reproduction number is denoted by $R_{0}$ and is defined as the expected number of people getting secondary infection among the whole susceptible population [13]. This number determines the potential for the spread of disease within a population. When $R_{0}<1$ each infected individual produces on average less than one new infected individual so that the disease is expected to die out. On the other hand if $R_{0}>1$ then each individual produces more than one new infected individual so that the disease is expected to continue spreading in the population. This means that the threshold quantity for eradicating the disease is to reduce the value of $R_{0}$ to less than one.

The basic reproductive number $R_{0}$ can be determined using the next generation matrix. In this method $R_{0}$ is defined as the largest eigenvalue of the next generation matrix. The formulation of this matrix involves classification of all compartments of the model in to two classes: infected and non-infected. That is, the basic reproduction number cannot be determined from the structure of the mathematical model alone but depends on the definition of infected and uninfected compartments.

Assume that there are $n$ compartments in the model and of which the first $m$ compartments are with infected individuals [3]. From the system (1) - (4) the first three equations are considered and decomposed into two groups: $F$ contains newly infected cases and $\mathrm{V}$ contains the remaining terms. Let $X=[S, A, I, V]^{t}$ be a column vector and the differential equations of the first three compartments are rewritten as $F(X)-V(X)$.

Now, let $F(X)=\left[\begin{array}{lll}F_{1}, & F_{2}, & F_{3}\end{array}\right]^{t}$. Here (i) $F_{1}=(\beta S A /$ $N$ ) denotes newly infected cases which arrive into the asymptomatic compartment, (ii) $F_{2}=0$ denotes newly infected cases arrived into the infectious compartment, and (iii) $F_{3}=0$ denotes newly infected case from susceptible compartment. Further, let $V(X)=\left[V_{1}, V_{2}, V_{3}\right]^{t}$. Here $V_{1}=a A, V_{2}=-\alpha A+b I$ and $V_{3}=-\delta A-\omega I+\mu V$. Here, as it is already mentioned, the parameters $a, b, c$ denote $a=\alpha+\mu+\theta+\delta-\eta, b=\omega+\mu+\rho-\emptyset, c=\mu+\gamma$.

The next step is the computation of square matrices $F$ and $V$ of order $m \times m$, where $m$ is the number of infected classes, defined by $F=\left[\partial F_{i}\left(E_{0}\right) / \partial x_{j}\right]$ and $V=$ $\left[\partial V_{i}\left(E_{0}\right) / \partial x_{j}\right]$ with $1 \leq i, j \leq m$, such that $F$ is nonnegative, $V$ is a non-singular matrices and $E_{0}$ is the disease free equilibrium point DFE.

If $F$ and $\mathrm{V}$ are non-negative and $V$ is non-singular then $V^{-1}$ is non-negative and thus $F V^{-1}$ is also non-negative. Also, the matrix $F V^{-1}$ is called the next generation matrix for the model. Finally, the basic reproduction number $R_{0}$ is given by $R_{0}=\rho\left(\mathrm{FV}^{-1}\right)$. In general, $\rho(A)$ denotes the spectral radius of matrix $A$ and the spectral radius is the biggest nonnegative eigenvalue of the next generation matrix.

The Jacobian matrices for $F(X)$ and $V(X)$ with respect to
$(A, \quad I, \quad V)$ can be constructed as

$$
J_{F}(X)=\left[\begin{array}{ccc}
\frac{\beta S}{N} & 0 & 0 \\
0 & 0 & 0 \\
0 & 0 & 0
\end{array}\right] J_{V}(X)=\left[\begin{array}{ccc}
a & 0 & 0 \\
-\alpha & b & 0 \\
-\delta & -\omega & { }_{c}
\end{array}\right]
$$

The Jacobian of $F$ and $V$ at the disease free equilibrium point $E_{0}$ takes the form respectively as

$$
J_{F}\left(E_{0}\right)=\left[\begin{array}{ccc}
\beta \mu_{d} / \mu & 0 & 0 \\
0 & 0 & 0 \\
0 & 0 & 0
\end{array}\right] J_{V}\left(E_{0}\right)=\left[\begin{array}{ccc}
a & 0 & 0 \\
-\alpha & b & 0 \\
-\delta & -\omega & c_{c}
\end{array}\right]
$$

It can be verified that the matrix $J_{V}\left(E_{0}\right)$ is non-singular as its determinant $\operatorname{det}\left[J_{F}\left(E_{0}\right)\right]=a b c$ is non-zero and after some algebraic computations its inverse matrix is constructed as

$$
\left[J_{V}\left(E_{0}\right)\right]^{-1}=\left[\begin{array}{ccc}
1 / a & 0 & 0 \\
\alpha / a b & 1 / b & 0 \\
(\alpha \omega+b \delta) /(a b c) & \omega / b c & 1 / c
\end{array}\right]
$$

The product of the matrices $J_{F}\left(E_{0}\right)$ and $\left[J_{V}\left(E_{0}\right)\right]^{-1}$ can be computed as

$$
\begin{gathered}
=\left[\begin{array}{ccc}
\beta \mu_{d} / \mu & 0 & 0 \\
0 & 0 & 0 \\
0 & 0 & 0
\end{array}\right]\left[\begin{array}{ccc}
\left.J_{F}\left(E_{0}\right)\right]\left[J_{V}\left(E_{0}\right)\right]^{-1} \\
(1 / a) & 0 & 0 \\
(\alpha / a b) & (1 / b) & 0 \\
(\alpha \omega+b \delta) /(a b c) & \omega / b c & (1 / c)
\end{array}\right] \\
=\left[\begin{array}{ccc}
\beta \mu_{d} / a \mu & 0 & 0 \\
0 & 0 & 0 \\
0 & 0 & 0
\end{array}\right]
\end{gathered}
$$

Now, it is possible to calculate the eigenvalues of the matrix $\left[J_{F}\left(E_{0}\right)\right]\left[J_{V}\left(E_{0}\right)\right]^{-1}$ to determine the basic reproduction number $R_{0}$ which is the spectral radius or the largest eigenvalue. Thus, the eigenvalues are computed by evaluating the characteristic equation $\operatorname{det}\left[\left[J_{F}\left(E_{0}\right)\right]\left[J_{V}\left(E_{0}\right)\right]^{-1}-\lambda I\right]=0$ or equivalently solving

$$
\left|\begin{array}{ccc}
\left(\beta \mu_{d} / a \mu\right)-\lambda & 0 & 0 \\
0 & -\lambda & 0 \\
0 & 0 & -\lambda
\end{array}\right|=0
$$

It reduces to the cubic equation for $\lambda$ as $\lambda^{2}\left[\left(\beta \mu_{d} / a \mu\right)-\right.$ $\lambda]=0$ giving the three eigenvalues as

$$
\lambda_{1}=\left(\beta \mu_{d} / a \mu\right), \lambda_{2}=0, \lambda_{3}=0
$$

However, the largest eigenvalue here is $\lambda_{1}=\left(\beta \mu_{d} / a \mu\right)$ 
and is the spectral radius or the threshold value or the basic reproductive number. Thus, it can be concluded that the reproduction number of the model is given by $R_{0}=$ $\left[\left(\beta \mu_{d}\right) /(\mu a)\right]$.

\subsection{Stability Analysis of the Disease Free Equilibrium}

In absence of the infectious disease, the model populations have a unique disease free steady state $E_{0}$. To find the local stability of $E_{0}$, the Jacobian method of the model equations evaluated at DEF $E_{0}$ is used. Also, to determine the global stability at $E_{0}$ M-matrix method given in [10] is used. It is already shown that the DFE of model (1) - (4) is given $\mathrm{b} E_{0}=\{\tau / \mu, 0,0,0\}$. Now, following [5-8] the stability analysis of DFE is conducted and the results are presented in the form of theorems and proofs in the following.

\subsubsection{Local Stability of Disease Free Equilibrium Point}

Theorem 1: The DFE $E_{0}$ of the system (1) - (4) is locally asymptotically stable if $R_{0}<1$ and unstable if $R_{0}>1$.

Proof: Consider the right hand side expressions of the equations (1) - (4) as functions so as to find the Jacobian matrix as follows:

$$
\begin{gathered}
d S / d t=\tau-(\beta A S / N)-\mu S \equiv g_{1}(S, \quad A, \quad I, \quad V) \\
d A / d t=(\beta A S / N)-(\alpha+\mu+\theta+\delta-\eta) A \\
\quad \equiv g_{2}(S, \quad A, \quad I, \quad V) \\
d I / d t=\alpha A-(\omega+\mu+\rho-\emptyset) I \equiv g_{3}(S, \quad A, \quad I, \quad V) \\
d V / d t=\delta A+\omega I-(\mu+\gamma) V \equiv g_{4}(S, \quad A, \quad I, \quad V)
\end{gathered}
$$

Now, the Jacobian matrix of $\left(g_{1}, g_{2}, g_{3}, g_{4}\right)$ with respect to $(S, A, I, V)$ is given by

$$
J=\left[\begin{array}{cccc}
-\mu-(\beta A / N) & -(\beta S / N) & 0 & 0 \\
(\beta A / N) & (\beta S / N)-a & 0 & 0 \\
0 & \alpha & -b & 0 \\
0 & \delta & \omega & -c
\end{array}\right]
$$

Furthermore, the Jacobian matrix $J$ of model at the disease free equilibrium $E_{0}$ reduces to

$$
J\left(E_{0}\right)=\left[\begin{array}{cccc}
-\mu & -\left(\beta \mu_{d} / \mu\right) & 0 & 0 \\
0 & \left(\left(\beta \mu_{d} / \mu\right)-a\right) & 0 & 0 \\
0 & \alpha & -b & 0 \\
0 & \delta & \omega & -c
\end{array}\right]
$$

Now, the eigenvalues of $J\left(E_{0}\right)$ are required to be found. The corresponding characteristic equation $\operatorname{det}\left[J\left(E_{0}\right)-\lambda I\right]=$ 0 is expanded and simplified as follows:

$$
\begin{aligned}
& \left|\begin{array}{cccc}
-\mu-\lambda & -\left(\beta \mu_{d} / \mu\right) & 0 & 0 \\
0 & \left(\left(\beta \mu_{d} / \mu\right)-a\right)-\lambda & 0 & 0 \\
0 & \alpha & -b-\lambda & 0 \\
0 & \delta & \omega & -c-\lambda
\end{array}\right|=0 \\
& -(\mu+\lambda)\left|\begin{array}{ccc}
\left(\left(\beta \mu_{d} / \mu\right)-a\right)-\lambda & 0 & 0 \\
\alpha & -b-\lambda & 0 \\
\delta & \omega & -c-\lambda
\end{array}\right|=0 \\
& (\mu+\lambda)(c+\lambda)(b+\lambda)\left[\lambda-\left(1-R_{0}\right) a\right]=0
\end{aligned}
$$

$$
(\mu+\lambda)(c+\lambda)(b+\lambda))\left[\left(1-R_{0}\right) a-\lambda\right]=0
$$

Thus, the four eigenvalues of the matrix $J\left(E_{0}\right)$ are determined as

$$
\begin{gathered}
\lambda_{1}=-\mu \\
\lambda_{2}=-c \\
\lambda_{3}=-b \\
\lambda_{4}=\left(R_{0}-1\right) a
\end{gathered}
$$

It can be observed that the first two eigenvalues $\lambda_{1}$ and $\lambda_{2}$ are absolutely negative quantities. However, the remaining two $\lambda_{3}$ and $\lambda_{4}$ are also negatives so long as the following restrictions on the parameters are valid: $a, b, c$ are positive and $R_{0}<1$.

Therefore, using $[1,10]$ it can be concluded that the DFE $E_{0}$ of the system of differential equations (1) - (4) is locally asymptotically stable if $R_{0}<1$ and unstable if $R_{0}>1$.

\subsubsection{Global Stability of Disease Free Equilibrium Point}

Theorem 2: The disease free equilibrium point $E_{0}$ of the model is globally asymptotically stable if $R_{0}<1$ and unstable if $R_{0}>1$.

Proof Using the comparison theorem the rate of change of the variables representing the disease classes of the model can be rewritten as

$$
\left[\begin{array}{c}
A^{0} \\
I^{0} \\
V^{0}
\end{array}\right]=(F-V)\left[\begin{array}{c}
A \\
I \\
V
\end{array}\right]-M \theta\left[\begin{array}{c}
A \\
I \\
V
\end{array}\right]
$$

Here in (16), $F$ and $V$ represent matrices at the disease free equilibrium point $E_{0}$ as

$$
\begin{gathered}
F=J_{F}\left(E_{0}\right)=\left[\begin{array}{ccc}
\beta \mu_{d} / \mu & 0 & 0 \\
0 & 0 & 0 \\
0 & 0 & 0
\end{array}\right] V=J_{V}\left(E_{0}\right) \\
=\left[\begin{array}{ccc}
a & 0 & 0 \\
-\alpha & b & 0 \\
-\delta & -\omega & c
\end{array}\right]
\end{gathered}
$$

Also $\theta$ is a non - negative matrix. However, $M=$ $\left[1-\left(S^{0} / N^{0}\right)\right]=0$ since $S^{0}=(\tau / \mu)$ and $N^{0}=(\tau / \mu)$. Therefore, the equation (16) reduces to the simplified form as

$$
\left[\begin{array}{l}
A^{0} \\
I^{0} \\
V^{0}
\end{array}\right] \leq(F-V)\left[\begin{array}{l}
A \\
I \\
V
\end{array}\right]
$$

Now, $(F-V)$ can be computed as

$$
\begin{array}{r}
F-V=\left[\begin{array}{ccc}
\beta \mu_{d} / \mu & 0 & 0 \\
0 & 0 & 0 \\
0 & 0 & 0
\end{array}\right]-\left[\begin{array}{ccc}
a & 0 & 0 \\
-\alpha & b & 0 \\
-\delta & -\omega & -c
\end{array}\right]= \\
{\left[\begin{array}{ccc}
\beta \mu_{d} / \mu-a & 0 & 0 \\
\alpha & -b & 0 \\
\delta & \omega & -c
\end{array}\right]}
\end{array}
$$

The eigenvalues of the matrix (17) are found by evaluating the characteristic equation $\operatorname{det}[(F-V)-\lambda I]=0$ as follows: 


$$
\begin{gathered}
\left|\begin{array}{ccc}
\left(\beta \mu_{d} / \mu-a\right)-\lambda & 0 & 0 \\
\alpha & -b-\lambda & 0 \\
\delta & \omega & -c-\lambda
\end{array}\right|=0 \\
(\beta-a-\lambda)(-b-\lambda)(-c-\lambda)=0 \\
{\left[\left(\beta \mu_{d} / \mu\right)-a\right]-\lambda=0,(-b-\lambda)=0,(-c-\lambda)=0} \\
\lambda_{1}=\left(\beta \mu_{d} / \mu\right)-a=\left(R_{0}-1\right) a, \lambda_{2}=-b, \lambda_{3}=-c
\end{gathered}
$$

Parametric expressions for the notations $a, b$ have been defined earlier. Here it can be observed that all the three eigenvalues $\lambda_{1}, \lambda_{2}, \lambda_{3}$ have negative real parts and hence the matrix is stable for $R_{0}<1$.

Therefore by the comparison theorem, it follows that $\{A, I, \quad V\} \rightarrow\{0,0,0\}$ and the remaining equations of model (1) - (4) give the solution $E_{0}=\{\tau / \mu, 0,0,0\}$. Thus, the system approaches to the DFE as time progresses i.e. $\{S, A, I, V\} \rightarrow E_{0}$ as $t \rightarrow \infty$. Hence, the disease free equilibrium point $E_{0}$ is globally asymptotically stable if $R_{0}<1$ and unstable if $R_{0}>1$.

\subsection{Stability Analysis of Endemic Equilibrium Point}

By definition it is true that at the endemic equilibrium point $E_{1}=\left\{S^{1}, A^{1}, I^{1}, V^{1}\right\}$ is the point where the disease persists or exists. To analyze the local stability of $E_{1}$, Jacobian of the model evaluated at that equilibrium point is used. Further, recall that the endemic equilibrium point $E_{1}=\left\{S^{1}, A^{1}, I^{1}, \quad V^{1}\right\}$ of the given model (1) - (4) is already computed. The local stability of endemic equilibrium point is stated and proved in Theorem 3 .

Theorem 3: The endemic equilibrium point is locally asymptotically stable if $R_{0}>1$.

Proof: The stability analysis of $E_{1}$ is conducted by following the similar procedure adopted as in the case of $E_{0}$. Thus, the procedure starts with the construction of Jacobian matrix at $E_{1}$. Now, the Jacobian matrix of the model given in (15) at endemic equilibrium point $E_{1}$ takes the form as

$$
\begin{aligned}
& S^{1}=\left(a \tau / \beta u_{d}\right)=\left(\tau / \mu R_{0}\right) \\
& A^{1}=\left(\mu \tau / \beta \mu_{d}\right)\left(R_{0}-1\right) \\
& I^{1}=\left(\alpha \mu \tau / b \beta \mu_{d}\right)\left(R_{0}-1\right) \\
& V^{1}=\left(\mu \tau / \beta \mu_{d}\right)[(\delta / c)+(\omega \alpha / c b)]\left(R_{0}-1\right) \\
& J=\left[\begin{array}{cccc}
-\mu-\left(\beta A^{1} / N\right) & -\left(\beta S^{1} / N\right) & 0 & 0 \\
\left(\beta A^{1} / N\right) & \left(\beta S^{1} / N\right)-a & 0 & 0 \\
0 & \alpha & -b & 0 \\
0 & \delta & \omega & -c
\end{array}\right] \\
& J\left(E_{1}\right)=\left[\begin{array}{cccc}
-R_{0} \mu & -a & 0 & 0 \\
\mu\left(R_{0}-1\right) & 0 & 0 & 0 \\
0 & \alpha & -b & 0 \\
0 & \delta & \omega & -c
\end{array}\right]
\end{aligned}
$$

Now the trace of $J\left(E_{1}\right)=-R_{0} \mu-b-c$ is a negative quantity while $\operatorname{det}\left(E_{1}\right)=\left(R_{0}-1\right)(a b c \mu)$ is a positive quantity provided that $a, b, c, \mu,\left(R_{0}-1\right)$ are positive quantities. Hence, using $[1,10]$ it can be concluded that the endemic equilibrium point $E_{1}$ is locally asymptotically stable if $R_{0}>1$.

\section{Numerical Simulation}

In this section, numerical simulation study of model equations (1) - (4) is carried out using the software MATLAB. To conduct the study, a set of physically meaningful values are assigned to the model parameters. These values are either taken from literature or assumed on the basis of reality. These sets of parametric values are given under figures.

The following have been observed in Figure 2: (i) initially the population size of susceptible compartment $S$ decreases. These cells get infected and migrate to asymptomatic compartment. At a later stage these susceptible cells increase because the production of new cells continues and the conversion of susceptible cells into asymptotic cells reduce due to treatment. (ii) Initially the population size of asymptomatic compartment $A$ increases because body immune system has not yet started fighting against the HIV virus and as a result the susceptible cells get infected and become asymptomatic and joins this compartment. (iii) As human immune system start fighting the asymptomatic cells began decreasing (iv) Initially the population size of asymptomatic compartment $A$ is increasing because body immune system has not yet started fighting the HIV virus.

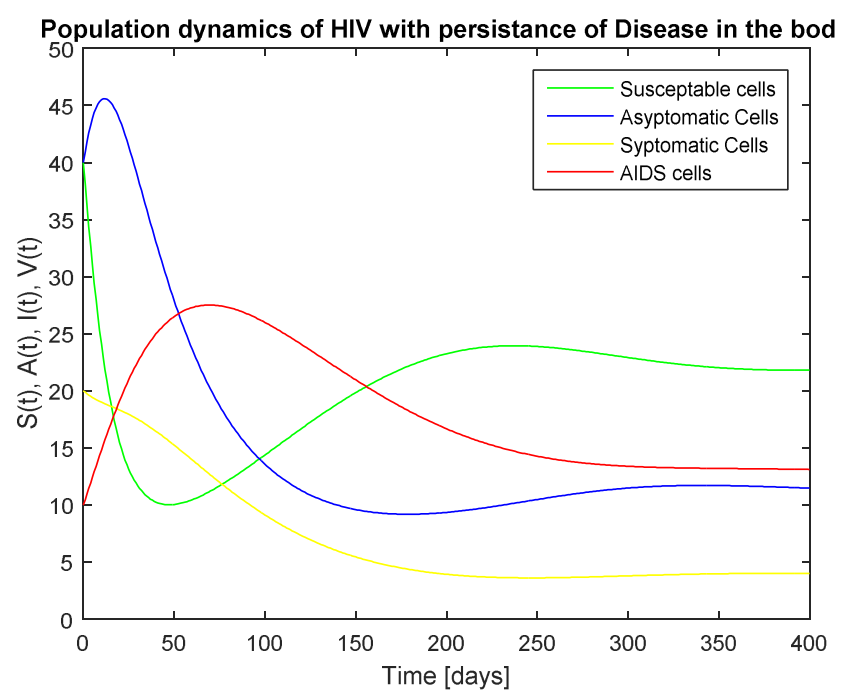

Figure 2. Population dynamics of SAIV compartments with the parametric values $\quad \tau=20, \mu=0.005, \beta=0.076, \alpha=0.008, \theta=0.01, \delta=$ $0.01, \eta=0.002, \omega=0.01, \rho=0.01, \phi=0.002$.

In figure 3 , it can be observed that the extinction of the virus for many days but finally the virus persists in human cells without showing any signs of infections. This happens because of treatment properly taken by the patients. The patient lives as normal as asymptomatic stage for long period of time.

The differences and similarities between the existing and modified model are given respectively in the tables 3 and 4 . 
Table 3. Differences of existing and modified model.

\begin{tabular}{lll}
\hline \multicolumn{2}{l}{ Differences } & \\
\hline SN & Existing model [11] & Modified (Present) model \\
\hline 1 & Has Three compartments & Has four compartments \\
2 & Has health cells, infected cells, and virus cells & Has susceptible cells, asymptomatic cells, symptomatic cells, AIDS cells \\
3 & Assumed only natural mortality & Assumed both death due to sickness and natural mortality \\
4 & Treatments not assumed & Assumed ART Treatment \\
5 & There is no treatment representation as parameter or variable & Treatment is taken as parameter \\
\hline
\end{tabular}

Table 4. Similarities of existing and modified model.

\begin{tabular}{l}
\hline Similarities \\
\hline Both existing [11] and modified (Present) model have the following similarities \\
Transmission rate \\
Natural mortality \\
Infected cell \\
\hline
\end{tabular}

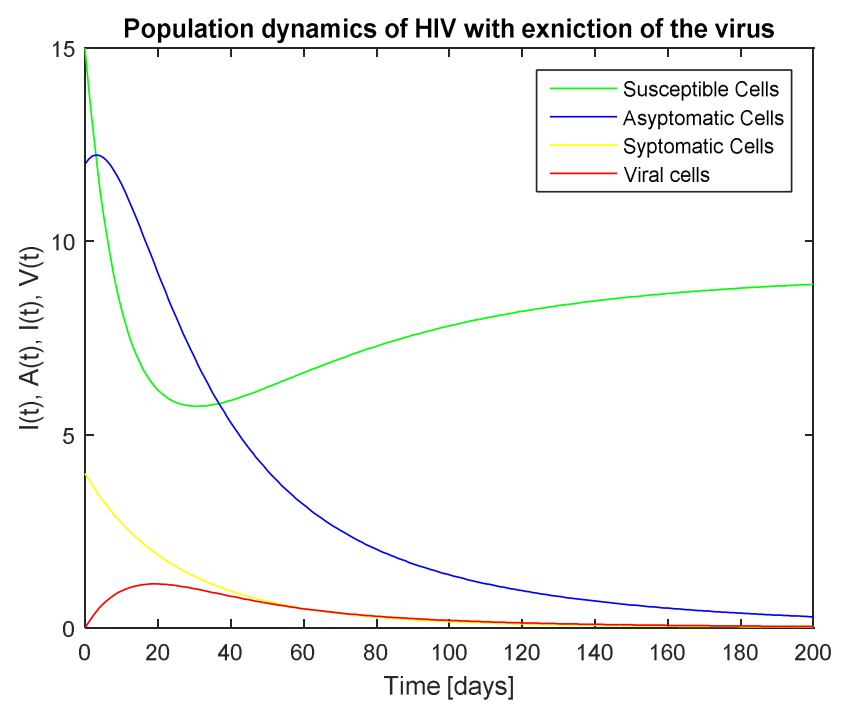

Figure 3. Population dynamics of SAIV compartments with the parametric values $\quad \tau=0.46, \mu=0.05, \beta=0.2, \alpha=0.008, \theta=0.01, \delta=$ $0.01, \eta=0.02, \omega=0.01, \rho=0.01, \phi=0.02 \mathrm{~F}$.

\section{Sensitivity Analysis}

Sensitivity analysis is used to determine the sensitivity of the model with respect to the parameters involved in it. That is, how changes in the value of the parameters of the model result in changing the dynamics of the infection. It is used to discover parameters that have a high impact on $R_{0}$ and should be targeted by intervention strategies. More precisely, sensitivity indices allow measuring the relative change in a variable when parameter changes [9]. If the result is negative, then the relationship between the parameters and $R_{0}$ is inversely proportional. In this case, the modulus of the sensitivity index will be taken so that the size of the effect of changing that parameter can be deduced.

On the other hand, a positive sensitivity index means that both the function and the parameter are proportional to each other i.e. both of them grow or decay together.

It is already shown that the explicit expression of $R_{0}$ is given by $R_{0}=\left[\left(\beta \mu_{d}\right) /(a \mu)\right]$. Since, $R_{0}$ depends only on four parameters, an analytical expression will be derived for its sensitivity to each of the parameters using the normalized forward sensitivity index as given by Chitnis [16].

$$
\begin{aligned}
& \Upsilon_{\beta}^{R_{0}}=\left[\partial R_{0} / \partial \beta\right] \times\left[\beta / R_{0}\right] \\
& \Upsilon_{\mu}^{R_{0}}=\left[\partial R_{0} / \partial \mu\right] \times\left[\mu / R_{0}\right] \\
& \Upsilon_{\eta}^{R_{0}}=\left[\partial R_{0} / \partial \gamma\right] \times\left[\gamma / R_{0}\right] \\
& \Upsilon_{\alpha}^{R_{0}}=\left[\partial R_{0} / \partial \gamma\right] \times\left[\gamma / R_{0}\right] \\
& \Upsilon_{\phi}^{R_{0}}=\left[\partial R_{0} / \partial \gamma\right] \times\left[\gamma / R_{0}\right] \\
& \Upsilon_{\theta}^{R_{0}}=\left[\partial R_{0} / \partial \gamma\right] \times\left[\gamma / R_{0}\right] \\
& \Upsilon_{\delta}^{R_{0}}=\left[\partial R_{0} / \partial \gamma\right] \times\left[\gamma / R_{0}\right]
\end{aligned}
$$

Table 5. Sensitivity of $R_{0}$ evaluated for the parametric values.

\begin{tabular}{ll}
\hline Parameter & Sensitivity index \\
\hline$\mu$ & +3.9409 \\
$\eta$ & -1.6553 \\
$\beta$ & +1 \\
$\alpha$ & -0.1379 \\
$\phi$ & -2 \\
$\theta$ & -0.1724 \\
$\delta$ & -0.1724 \\
\hline
\end{tabular}

From Table 5, it can be observed that the values of two parameters $\mu, \beta$ are positive sensitivity indices and values of the remaining five parameters $\eta, \alpha, \phi, \theta, \delta$ get negative sensitivity indices.

As it is observed from the table the parameter with large magnitude is $\mu$. Hence, it can be conclude that $\mu$ is the most sensitive parameter in the model equations. On the other hand an increase in these positive parameter values will cause an increasing $R_{0}$ this implies that disease persist in human cells. Similarly, a decrease in negative parameter values will cause a decrease in $R_{0}$ which means the disease die out from human cells.

\section{Result and Discussion}

In this study, a mathematical model describing the dynamics of Human Immunodeficiency Virus (HIV) with treatment by ART is formulated and analyzed. The model is 
developed based on biologically reasonable assumptions made about Human Immunodeficiency Virus (HIV) and its treatment. The mathematical analysis has shown that if the reproduction number $R_{0}<1$ then the disease free equilibrium point is locally and globally asymptotically stable implying that the disease wipes out and the treatment is successful which is supported by the simulation results given in Figure 2. Also, if $R_{0}>1$ then the disease free equilibrium point is unstable implying that the treatment is not successful. These theoretical results have been supported by the simulation study as it is shown in Figure 1. Furthermore, the endemic equilibrium point is stable if $R_{0}>1$ resulting that the infectious cells continue to replicate. This fact has also been supported by Figure 1.

\section{Conclusion}

In this study, a mathematical model of Human Immunodeficiency Virus HIV using ART as treatment has been formulated. Moreover, existence, positivity and boundedness of the formulated model are verified to illustrate that the model is biologically meaningful and mathematically well posed. In particular, the stability analyses of the model were investigated using the basic reproduction number and Routh Hurwitz criterion. Also, the solution of the model equations is numerically simulated and sensitivity analysis of the model is conducted. Furthermore, results of the research work presented in this paper reveal that the model formulated here effectively supports treatment for HIV disease.

\section{Acknowledgements}

The authors would like to thank the editor and the anonymous reviewers of the journal IOSR-JM for their helpful suggestions and remarks.

\section{References}

[1] Brauer F., P. van den Driessche and W. Jianhong. Mathematical Epidemiology, volume 1945. Springer-Verlag Berlin Heidelberg, Canada, 2008.
[2] W. S. Ronald and H. James. Mathematical biology: An Introduction with Maple and Matlab. Springer Dordrecht Heidelberg, Boston, (1996).

[3] J. Robertson. World Health Organization. AIDS epidemic update: November in 2009 UNAIDS/09.36E / JC1700E. ISBN 9789291738328 .

[4] A. A. Ejigu. Mathematical Modeling of HIV/AIDS transmission under treatment structured by age of infection, Stellenbosch University (2010).

[5] Alemu Geleta Wedajo, Boka Kumsa Bole, Purnachandra Rao Koya. The Impact of Susceptible Human Immigrants on the Spread and Dynamics of Malaria Transmission. American Journal of Applied Mathematics. Vol. 6, No. 3, 2018, Pp. 117127. doi: 10.11648/j.ajam.20180603.13.

[6] Alemu Geleta Wedajo, Boka Kumsa Bole, Purnachandra Rao Koya. Analysis of SIR Mathematical Model for Malaria disease with the inclusion of Infected Immigrants. IOSR Journal of Mathematics (IOSR-JM). Volume 14, Issue 5 Ver. I (Sep - Oct 2018), PP 10-21 DOI: 10.9790/5728-1405011021.

[7] Geremew Kenassa Edessa, Boka Kumsa, Purnachandra Rao Koya. Dynamical behavior of Susceptible prey - Infected prey - Predator Populations. IOSR Journal of Mathematics (IOSRJM). Volume 14, Issue 4 Ver. III (Jul - Aug 2018), PP 31-41. DOI: $10.9790 / 5728-1404033141$.

[8] Solomon Tolcha, Boka Kumsa, Purnachandra Rao Koya. Modeling and Simulation Study of Mutuality Interactions with Type II functional Response and Harvesting. American Journal of Applied Mathematics. Vol. 6, No. 3, 2018, pp. 109116. doi: 10.11648/j.ajam.20180603.

[9] Chitnis N., Hyman J. M., and Cushing J. M. (2008). Determining important Parameters in the spread of malaria through the sensitivity analysis of a mathematical Model. Bulletin of Mathematical Biology 70 (5): 1272-12.

[10] P. van den Driesch and James Warmouth. Reproduction numbers and sub-threshold endemic equilibria for compartmental models of disease transmission. Mathematical Biosciences 180 (2002) 29-48.

[11] Jones E., P., Roemer. Department of Mathematics United States Naval Academy, 572C Holloway Road, Chauvenet Hall, Annapolis, MD 21402 (peteusna@gmail.com, raghupat@usna.edu). 